\title{
THE IMPACT OF THE TIRAD SYSTEM ON THE MEDICAL TACTICS IN PATIENTS WITH THYRIOD NODULES
}

\section{A.Ametov,}

Y.Aleksandrov, ${ }^{2}$

E.Yanovskaya ${ }^{2}$

\section{Correspondence:}

YuriAleksandrov, Department of Surgery of Yaroslavl State Medical University,

Russia, 150000, Yaroslavl, Revolutionnaya, 5 Tel.: +7(4852)30-56-41 Fax:+7(4852)72-91-42 Email:yka2000@mail.ru

1. Russian Medical academy for advance medical studies.

2. Department of Surgical Diseases of Pediatric Faculty of Yaroslavl State Medical University, Yaroslavl, Russia;

\section{INTRODUCTION}

Widespread use of ultrasound has led an increase of patients with thyroid nodules. But individual scores of ultrasound images leads to different estimates of the results and the misunderstandings between specialists. A decision of problem is possible through standardization based on TIRADS.

\section{PURPOSE}

Evaluated the impact the application of TIRADS and TBSRTC on medical assistance to patients with thyroid nodules.

\section{MATERIALS and METHODS}

The examination dates of 4415 people was estimated retrospectively. In group A (3370 people) the FNAB was done in all nodules more $10 \mathrm{~mm}$. In group B (1045 people) selection of patients for FNAB was performed into account TIRADS. The results of FNAB was evaluated for TBSRTC.

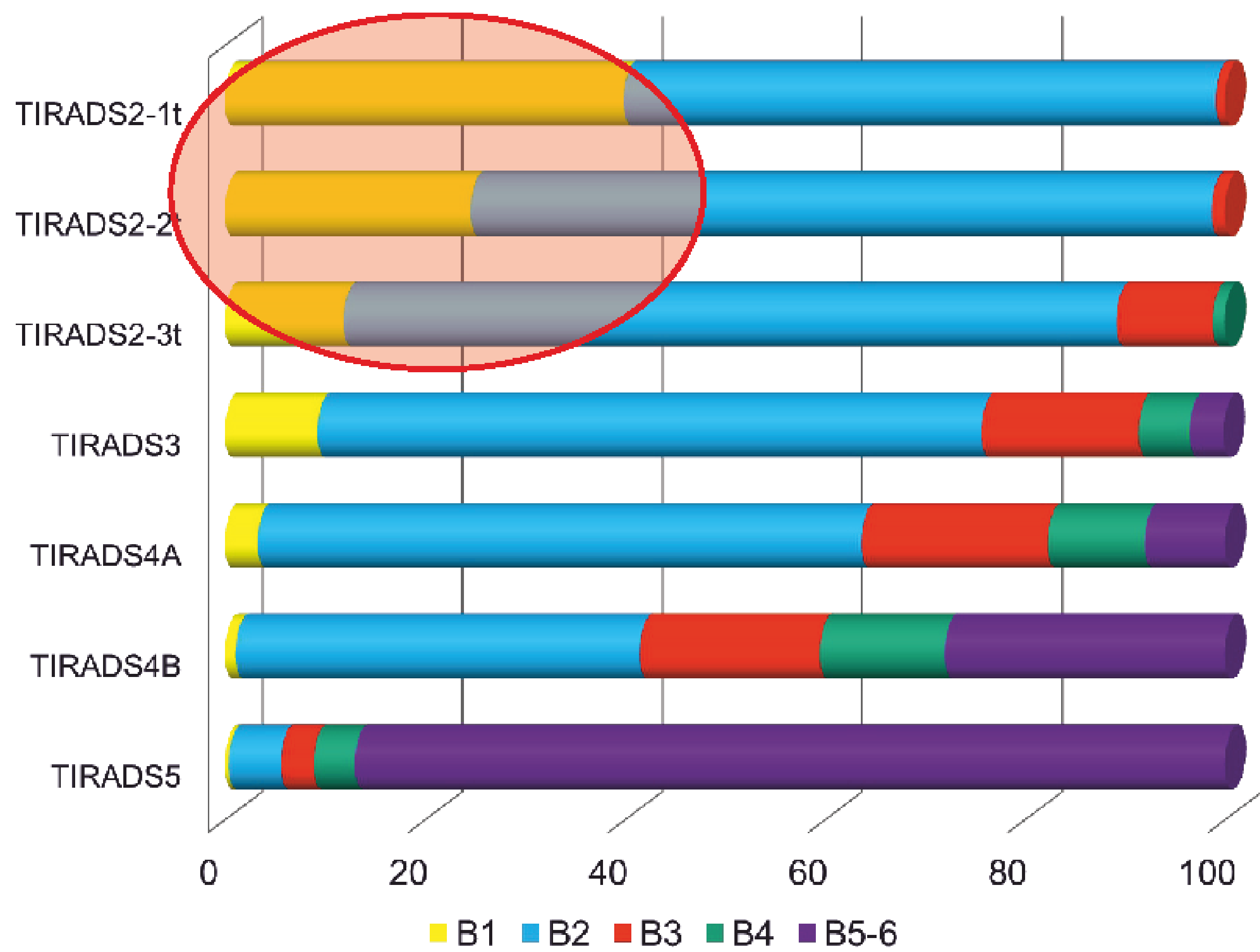

Pic. 6. Cases in which repeated FNAB do not need to

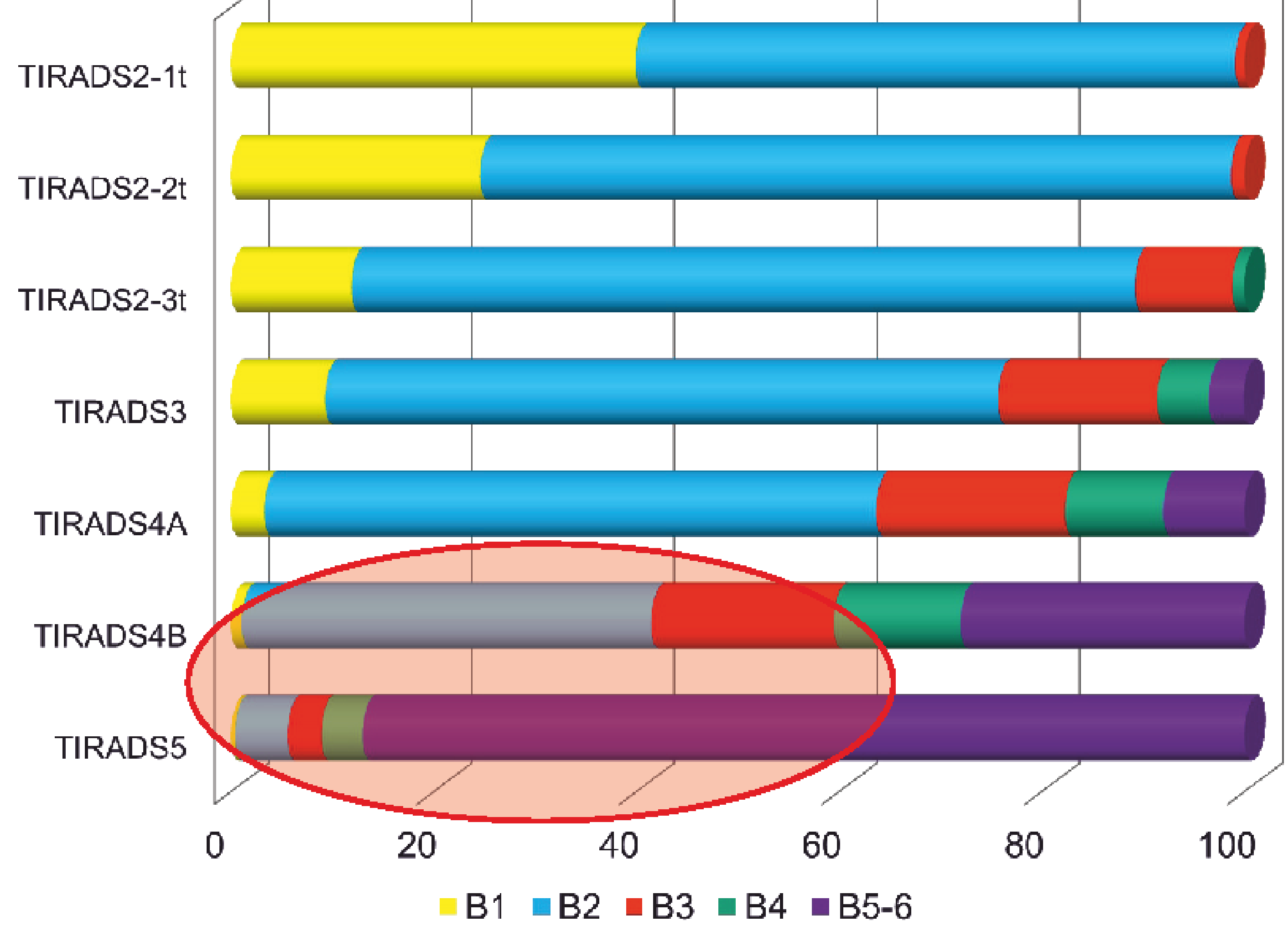

Pic.7. Cases that require repeated biopsies

\section{CONCLUSION}

Most patients with TIRADS2 does not require FNAB. The patients with TIRADS2 needs for FNAB necessarily despite previous conclusions excluding cancer. The patients with TIRADS4-5 have high risk of thyroid cancer therefore they need of the periodical control (FNAB). TIRADS makes more specific evidence for FNAB, limits the number of people who needs of FNAB and sends cytologist at the probability of detecting thyroid tumors. TIRADS governs assistance to patients with thyroid nodules.

\section{RESULTS}

Results: conclusions in group A were received: TBSRTC1 in $32,9 \%$, TBSRTC2 - 57,2\%, TBSRTC3,4 - 4,2\%, TBSRTC5 $2,6 \%$, TBSRTC6 $-3,1 \%$. Conclusions in group B were different: TBSRTC1 - 3,4\%, TBSRTC2 - 85,8\%, TBSRTC3,4-4,5\%, TBSRTC5 - 2,7\%, TBSRTC6 - 3,6\%. Results were connected with indications for FNAB and motivation of specialists who performed investigation. The number of not sufficiently informative results in group A was $32,9 \%$, in group B - 3,4\%. Widespread use of FNAB (account recommendations) has led to increase of benign conclusions (TBSRTC2): $57,2 \%$ (group A) \& $85,8 \%$ (group B). Detection of thyroid cancer in both groups was similar: $3,1 \%$ (A) \& 3,6\% (B). In group B in categories TIRADS4-5 frequency of detection of tumors was $62,1 \%$, including cancer $54,1 \%$. In categories TIRADS1-3 frequency of detection tumor was $8,1 \%$, including cancer $-1,8 \%$.

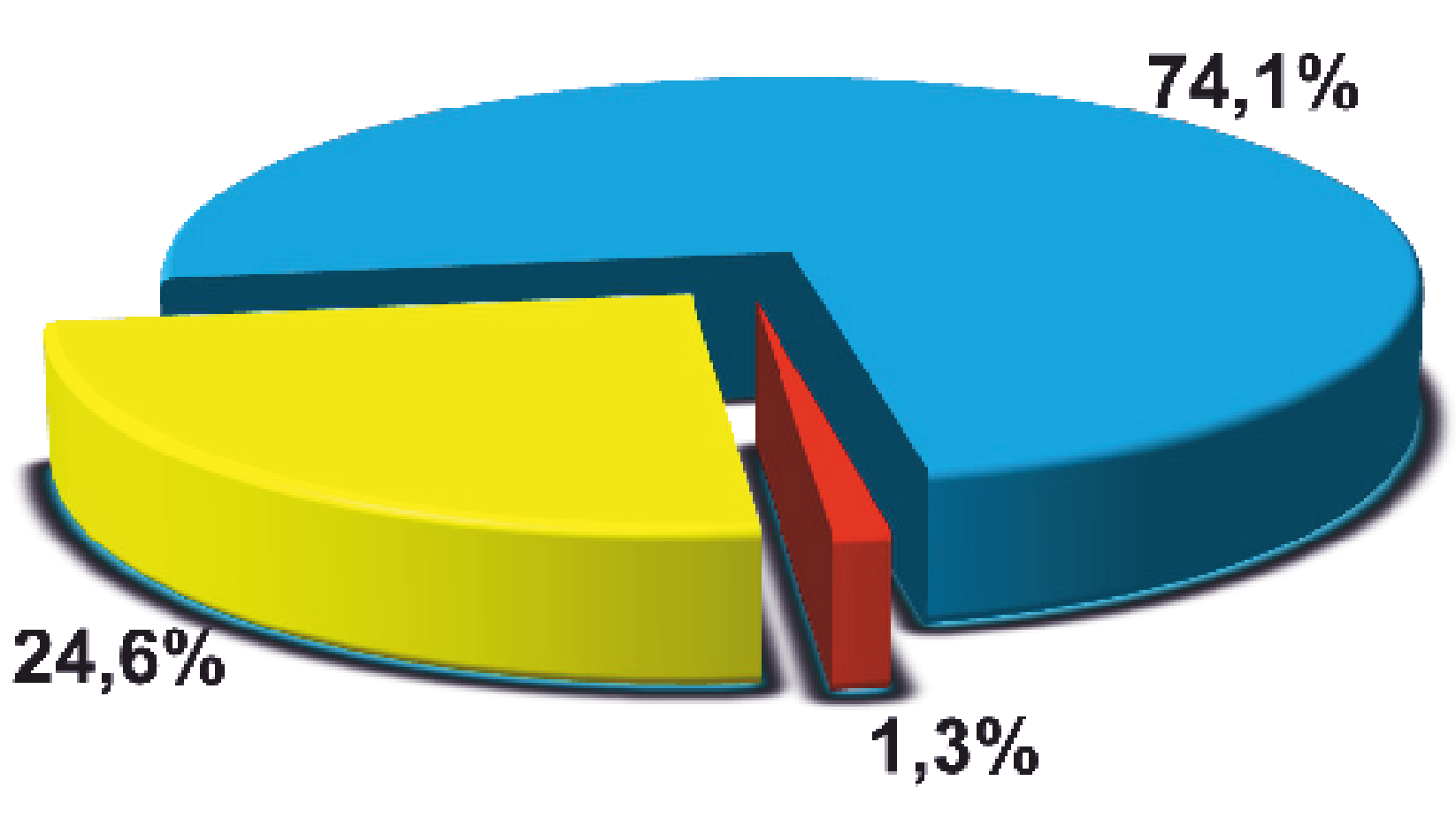

Pic.1. The results of FNAB for TIRADS 2

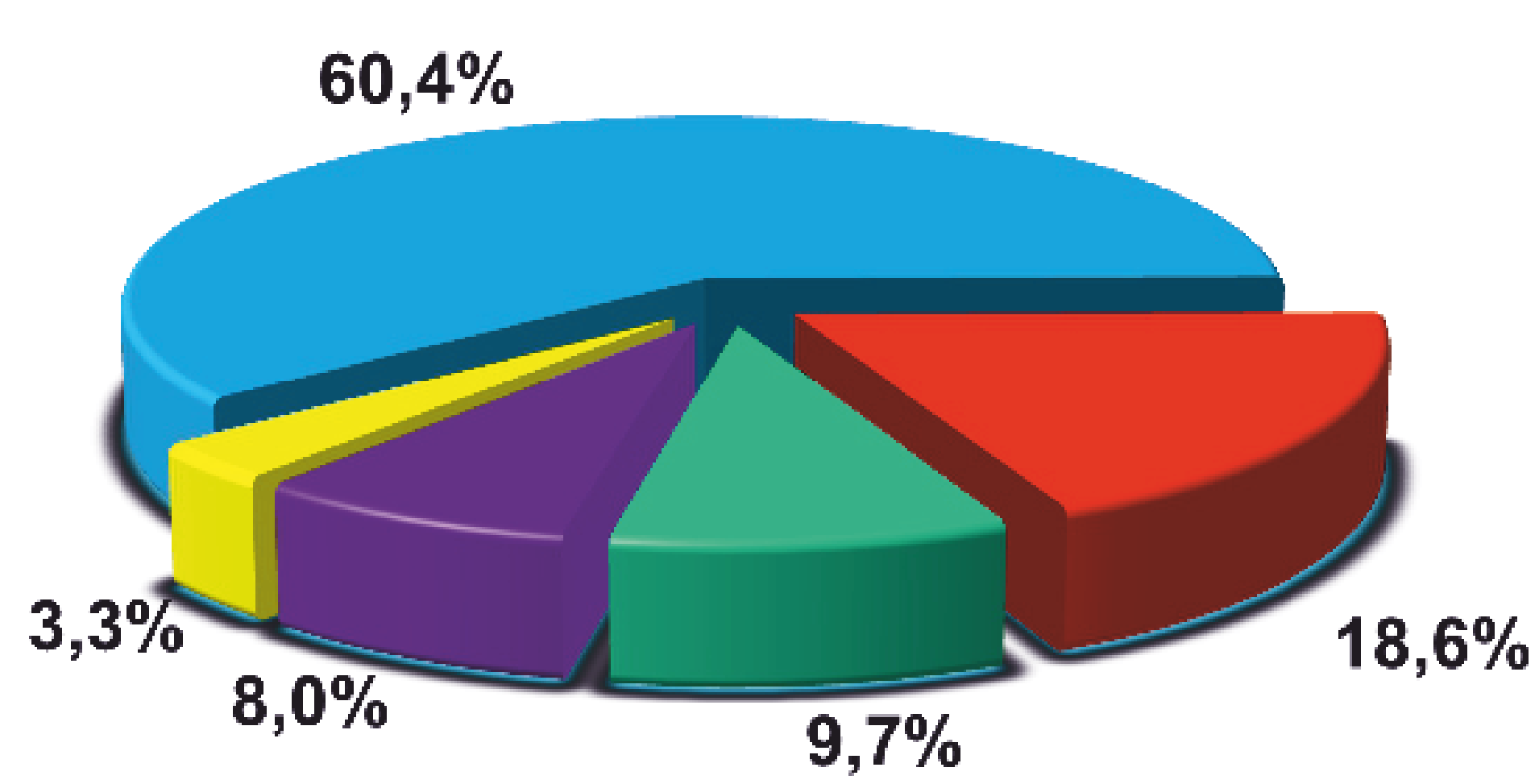

Pic.3. The results of FNAB for TIRADS4A

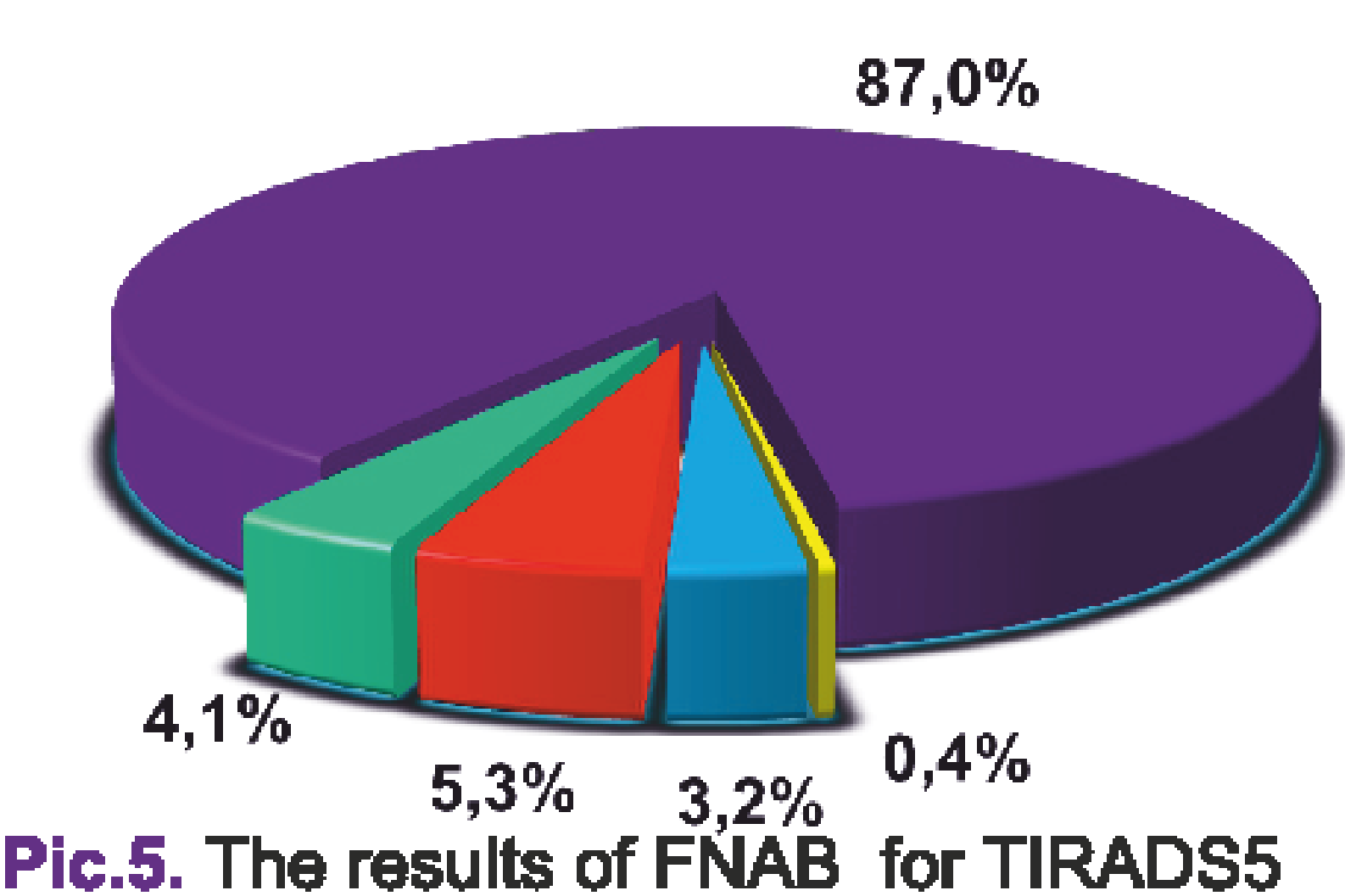

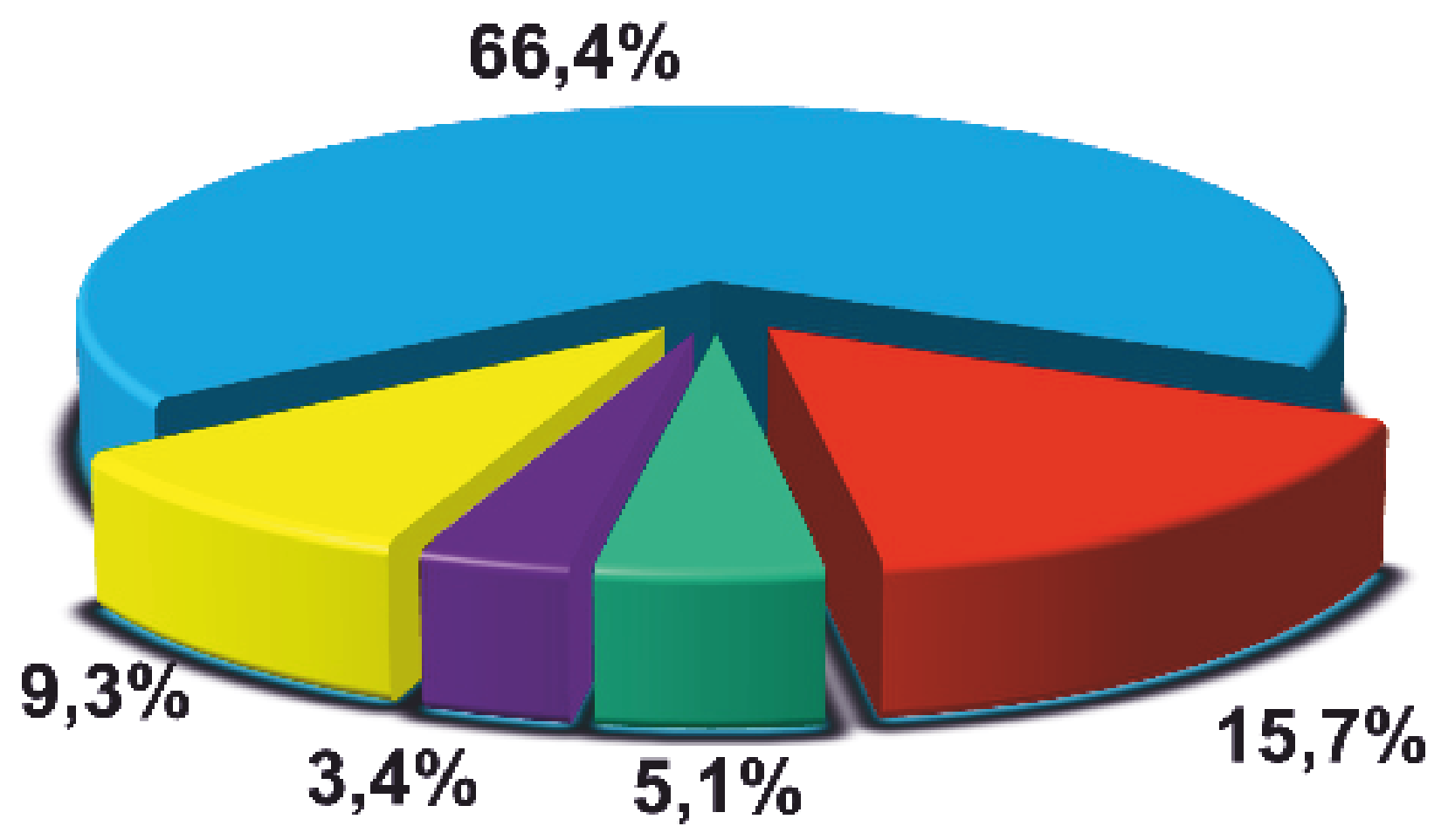

Pic.2. The results of FNAB for TIRADS3

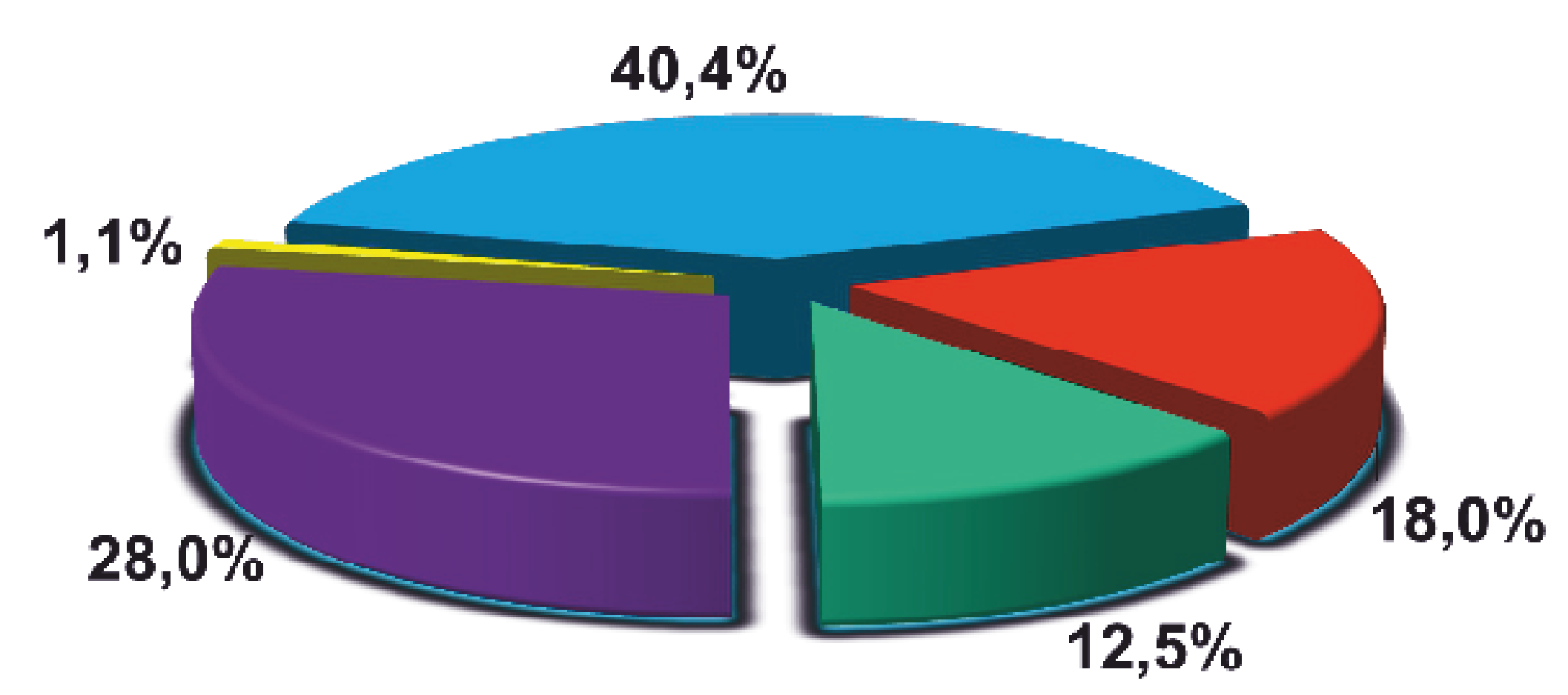

Pic.4. The results of FNAB for TIRADS4B

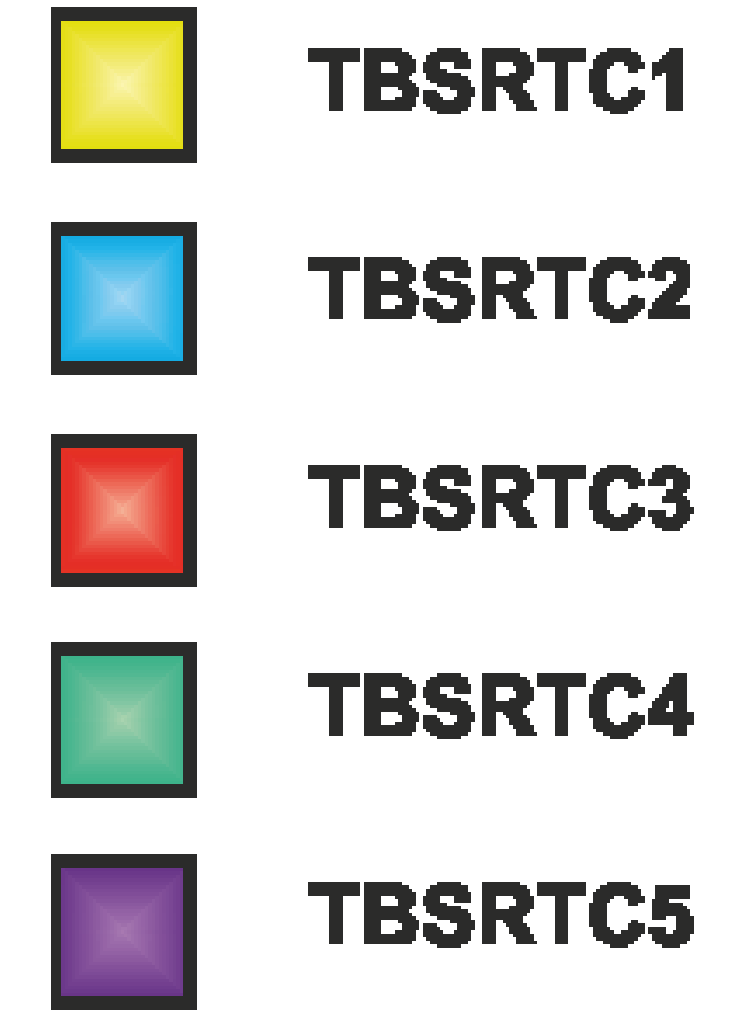

